[J. Jpn. Soc. Starch Sci., Vol. 33, No. 1, p. 71 79 (1986)]

Award Address

\title{
35 Years of Research in Glycogen Metabolism
}

\author{
Joseph LARNER \\ Department of Pharmacology, University of Virginia, School of Medicine \\ (Charlottesville, Virginia 22908, U.S.A.) \\ Received November 15, 1985
}

\section{INTRODUCTION AND HISTORICAL}

I began my scientific work for the $\mathrm{Ph}$. $\mathrm{D}$. degree in St. Louis under the late Gerty and Carl Cori by discovering the action of the metabolic debranching enzyme termed amylo-1,6glucosidase. This enzyme which cleaves the $\alpha-1,6$ branch linkages in glycogen and amylopectin was characterized initially through the identification of its reaction product, free glucose. Amylo-1, 6-glucosidase was then used together with the previously known enzyme phosphorylase in 2 ways, either together to measure the $\%$ of branch points (or average chain length) in glycogen and amylopectin; or sequentially to determine the arrangement of the branch points in the molecule. From these latter studies we deduced an irregularly branched rather than a regularly branched structure for these polysaccharides (Fig. 1)., ${ }^{1,2}$

It was a great source of personal pleasure when the late Professor Ziro Nikuni came to visit my laboratory in Urbana, Ill. to discuss these results. A friendship was immediately formed and as a result 2 scientists, Dr. Susumu Hizukuri and Yasuhito Takeda from Osaka and Kagoshima came to work in my laboratory in Cleveland and Charlottesville respectively. They both did outstanding work on another new enzyme glycogen synthase, its regulation and its chemical characterization. I in turn was invited to Japan and spent a memorable 3 month period in Kagoshima in 1975 . There I performed studies on glycogen synthase using defined sugars and oligosaccharides as primers for initiating glycogen synthesis de novo. ${ }^{32}$ As you can see, I have had close ties with Japan and Japanese scientists for over 20 years. Most recently, I have had 2 Japanese scientists from Sendai sent by Dr. Tsuiki working in my laboratory in Charlottesville. These have been Dr. Kunimi Kikuchi and Shinri Tamura who have worked in the general area of insulin action and its effect to activate glycogen synthase. In 1984, Dr. Hizukuri visited my laboratory in Charlottesville in connection with his participating in an American Chemical Society Symposium honoring the late Dexter French. He delighted me at that time by pointing out that in his most recent work he and his colleagues had utilized a new elegant enzymatic method for measuring average chain length. ${ }^{4)}$ With this new method they had determined (in 1983) a value of 18.5 for wheat amylopectin, essentially identical to the value I had determined in 1952 for the same substance. ${ }^{2)}$ Needless to say, I was pleased. My aim today is to review the past 35 years of work on glycogen metabolism and its control in terms of both the covalent control by phosphorylation and the allosteric control by soluble factors. I will also discuss hormonal control exerted on covalent phosphorylation via second meassengers.

\section{ENZYMOLOGY OF GLYCOGEN SYNTHESIS AND DEGRADATION-OVERALL ASPECTS}

The overall enzymatic pathway for glycogen metabolism is now well understood. As shown in Fig. 2, UDPG is synthesized from glucose 1-P and UTP by the enzyme UDPG pyrophos- 


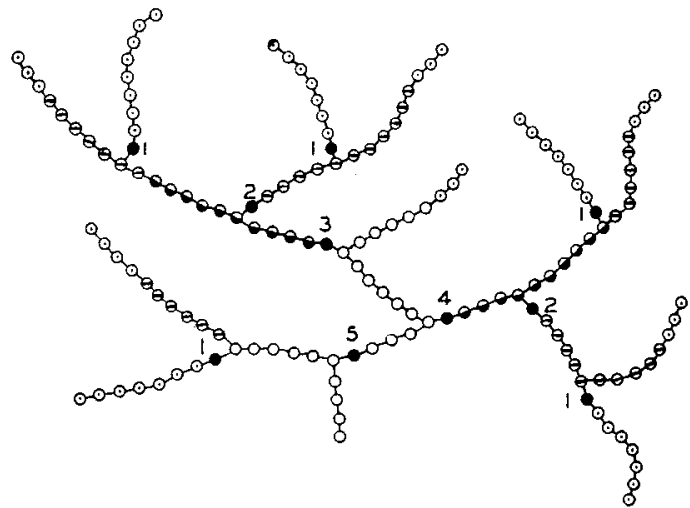

Fig. 1. Model of segment of muscle glycogen based on results obtained by stepwise enzymatic degradation.

$\odot, \Theta$, and $\ominus$ glucose residues removed by first, second, and third degradation with phosphorylase, respectively. - glucose residues removed by amylo-1, 6-glucosidase. Of five tiers three were degraded, corresponding to 122 out of 150 glucose residues.

Reproduced from Ref. 2).

\section{Synthesis}

\section{Degradation}

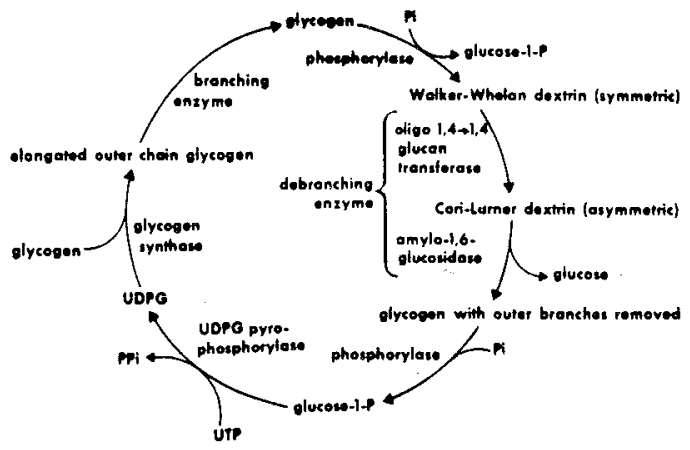

Fig. 2. The glycogen cycle.

The enzymes which catalyze the synthesis and degradation of the $\alpha-1,4$ and $\alpha-1,6$ linkages are shown.

Reproduced from J. LARNER: in Enzyme Therapy in Genetic Diseases IX, Birth Defects, Original Article Series, D. Bergsma ed., Williams and Wilkins Comp., Baltimore, p. 149 (1973).

phorylase ${ }^{5)}$ with inorganic pyrophosphate $\left(\mathrm{PP}_{\mathrm{i}}\right)$ as the other reaction product. UDPG is then used as the direct glucosyl donor by the rate limiting enzyme of synthesis, glycogen synthase to elongate the outer chains of glycogen. ${ }^{6)}$ Once the outer chains have become sufficiently elongated ( $8-11$ residues) the branching enzyme

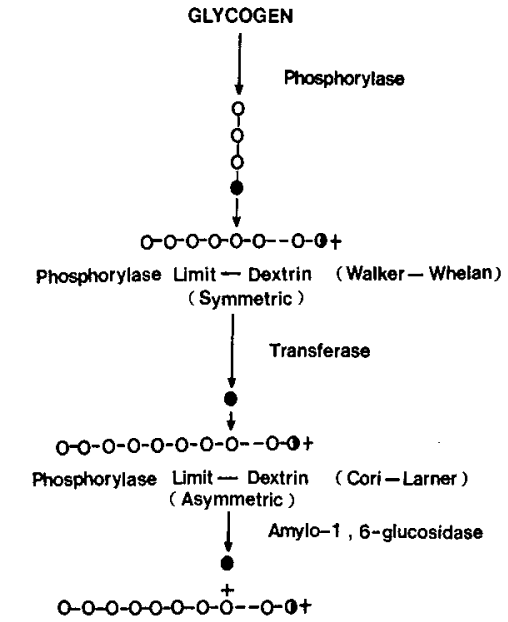

Fig. 3. Enzymatic debranching of glycogen as mediated by amylo-1, 6-glucosidase, transferase (oligo- 1,4 $\rightarrow 1,4$-glucantransferase) in conjunction with phosphorylase.

$\bigcirc$, -glucose;, reducing glucose unit; $\bigcirc-, 1,4$-bond; , 1,6-bond; , 1, 6 glucose.

Reproduced from M. ABDULLAH, P. M. TAYLOR and W. J. WHELAN: in Ciba Foundation Symposium-Control of Glycogen Metabolism, J. \& A. Churchill Ltd., London, p. 123 (1964).

amylo-1, $4 \rightarrow 1,6$-transglucosylase then inserts the branch points by a transfer reaction in which an $\alpha-1,6$ link is formed and an $\alpha-1,4$ link is broken. Thus both the 1,4 and 1,6 linkages of glycogen are formed. ${ }^{7}$

In glycogen degradation, the chief rate limiting enzyme is phosphorylase. Although catalyzing a reversible reaction in vitro, in the cell, it is driven toward degradation by the high millimolar concentration of inorganic $\mathrm{P}_{\mathrm{i}}$ (3-5 $\mathrm{mM}$ ) compared to the very low micromolar concentration of glucose 1-P $(10-20 \mu \mathrm{M})$. At phosphorylase equilibrium, the ratio of $\mathrm{P}_{\mathrm{i}} /$ glucose $1-\mathrm{P}$ is $3 / 1$, but in the cell the ratio is $300 / 1$. Finally, the debranching enzyme, which contains two separate catalytic activities within a single polypeptide chain rearranges the symmetric Walker-Whelan phosphorylase limit dextrin (Fig. 3) to the asymmetric Cori-Larner limit dextrin (Fig. 4) and then cleaves the exposed $\alpha-1,6$ linked glucose residue to free glucose. Thus, during metabolic degradation of glycogen by the 2 enzymes phosphorylase and debranching enzyme, over $90 \%$ glucose $1-\mathrm{P}$ and less 


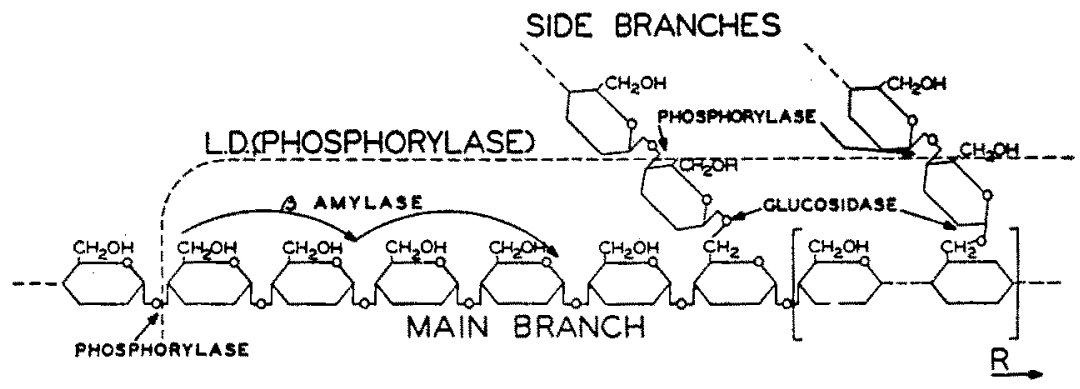

Fig. 4. Structural model of a portion of a branched polysaccharide showing the sites of enzymatic action.

LD corresponds to the limit dextrin formed by exhaustive action of phosphorylase. $R$ refers to the reducing end.

Reproduced from Ref. 1).

than $10 \%$ free glucose are produced. The free glucose arises specifically from hydrolysis of the branch linkages.

To summarize, there are 2 separate enzyme pathways catalyzing the synthesis and degradation of the 2 major linkage types in glycogen -a total of 4 enzymes for synthesis and degradation.

\section{BIOCHEMICAL CONSIDERATIONS}

Let us examine these 2 pathways more closely as a biochemical unit. For each turn of the cycle (Fig. 2) made up of these 2 pathways if we sum up these reactions, we can determine that a molecule of UTP is utilized in what might be turned a glycogen dependent UTP' ase. ${ }^{8)}$ Thus,

(1) Glucose $1 \cdot \mathrm{P}^{-2}+\mathrm{UTP}^{-4}$

$$
\Longrightarrow \mathrm{UDPG}^{-2}+\mathrm{PP}_{\mathrm{i}}^{-4}
$$

(2) $\mathrm{UDPG}^{-2}+$ glycogen

(3) Glycogen $+\mathrm{P}_{\mathrm{i}}{ }^{-2}$

$$
\longrightarrow \mathrm{UDP}^{-3}+\text { glycogen }+1+\mathrm{H}^{+}
$$

$$
\rightleftharpoons \text { glucose } 1-\mathrm{P}^{-2}+\text { glycogen }-1
$$

(4) $\mathrm{PP}_{\mathrm{i}}^{-4}+\mathrm{H}_{2} \mathrm{O} \longrightarrow 2 \mathrm{P}_{\mathrm{i}}^{-2}$

SUM $\mathrm{UTP}^{-4}+\mathrm{H}_{2} \mathrm{O} \longrightarrow \mathrm{UDP}^{-3}+\mathrm{P}^{-2}+\mathrm{H}^{+}$ As in any $\mathrm{ATP}^{\prime}$ ase reaction, a proton is produced to neutralize the unit negative charge formed.

A comparison of the nucleotide sugar UDPG and the sugar phosphate, glucose 1-P is of interest. It is clear that the nucleotide sugar which is simply an enlarged sugar phosphate derivative is a much more efficient glucosyl donor. Why is this so? If we compare the 2 molecules and the 2 transfer reactions we can readily see the answer. Glucose 1-P is a phosphomonoester. As a result, when the glucosyl moiety is transferred to glycogen, no unit charge difference occurs during the reaction (Eq. (3)). On the other hand, the nucleotide sugar is a phosphodiester, and when the glucosyl moiety is transferred, a proton is released (Eq. (2)).

- The free energy of neutralization of this proton is a powerful force driving the reaction to completion. For this reason, the nucleotide sugar is a much more efficient glucosyl donor than the sugar phosphate itself, a marvellous development of nature designed for increased efficiency in synthesis.

\section{COVALENT AND NON COVALENT CONTROL}

The two rate limiting enzymes of glycogen synthesis and degradation, namely, glycogen synthase and phosphorylase, are both under covalent and allosteric control. ${ }^{83}$ These controls have now been worked out and are rather well understood. They constitute a beautifully integrated system responsive to hormonal and other stimuli which signal either glycogen synthesis or glycogen degradation depending on the needs of the cell.

Let us first discuss control by covalent phosphorylation. Covalent phosphorylation in general has opposite effects on the 2 enzymes. Phosphorylase is activated while glycogen synthase is inactivated by covalent phosphorylation. 
Thus, under conditions where ATP is utilized for control, glycogen degradation would be favored and synthesis disfavored. Conversely, when ATP is not utilized for control, glycogen synthesis would be favored and degradation disfavored. The importance of this mechanism is that at the same time one enzyme can be turned on while the other is turned off, and vice versa. This dual enzyme control is the mechanism that ensures directionality of glucosyl carbon flow into or out of glycogen in an absolute manner when the cell needs to draw on its glycogen reserve or when it needs to conserve glucose as glycogen.

Now let us discuss the allosteric control. AMP is the most important allosteric activator of phosphorylase $b$, the inactive dephosphorylated form. Glucose 6-P is the most important activator of glycogen synthase $D$, the inactive

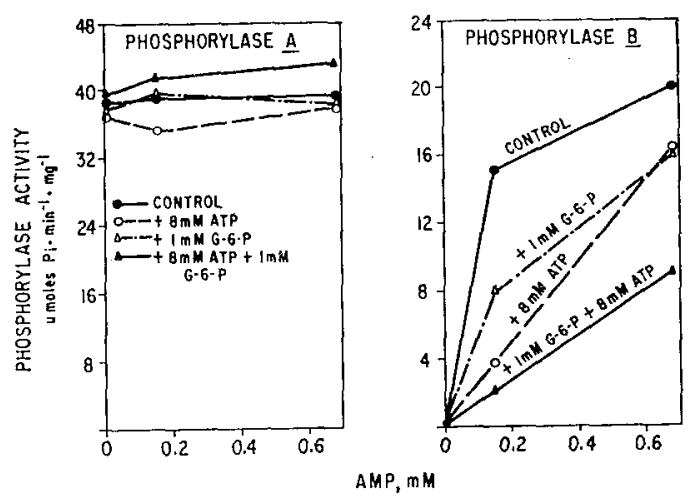

Fig. 5. Effect of ATP ( $8 \mathrm{mM})$ and G-6-P $(1 \mathrm{mM})$ on phosphorylase $a$ and $b$ activity at various levels of $5^{\prime}$-AMP.

Enzyme activity was assayed in glycerophosphate $(17 \mathrm{mM}), \operatorname{EDTA}(0.5 \mathrm{mM})$, cysteine $(15 \mathrm{mM})$, and glycogen $(1 \%), \mathrm{pH}$ 6. 8. Phosphorylase $b, 4 \times$ crystallized, was prepared from rabbit muscle and treated with Norit a prior to use. Phosphorylase $a, 10 \times$ crystallized, was prepared from a second phosphorylase $b$ preparation by the phosphorylase $b$ kinase reaction, recrystallized, and treated with Norit $a$. The aerobic AMP level in the perfused heart was approximately $0.2 \mathrm{mM}$ and rose to approximately $0.8 \mathrm{mM}$ after $2 \mathrm{~min}$ of anoxia. The aerobic concentration of ATP was approximately $8 \mathrm{mM}$.

Reproduced from H. E. MORGAN and A. PARMEGGIANI: in Ciba Foundation Symposium-Control of Glycogen Metabolism, J. \& A. Churchill Ltd., London, p. 254 (1964). phosphorylated form. Other activators are UDP glucose and $\mathrm{Mg}^{2+}$. On the other hand, ATP and glucose 6-P are important inhibitors of phosphorylase $b$ activation by AMP. As shown in Fig. 5, these molecules competitively inhibit the activation of phosphorylase $b$ by AMP but have no effect on phosphorylase $a$. For glycogen synthase $D, A T P, A D P, A M P, U D P$ and $P_{i}$ serve a similar function. They inhibit its activation by glucose 6-P. Taking this a step further, we can generalize that the inactive forms of both enzymes are in fact inactive when tested in an artificial mixture of metabolites made up of both allosteric activators and inhibitors at concentrations which approximate those in the cell milieu. In contrast, the active forms of the two enzymes namely, phosphorylase $a$, and glycogen synthase $I$ are still active in the artificial cell milieu mixture. Thus, inactive forms of both the enzyme, which are active in vitro when the allosteric activators are added alone, are in fact inactive when all of the cell constituents are present. The allosteric inhibitors can be thought of as competing away

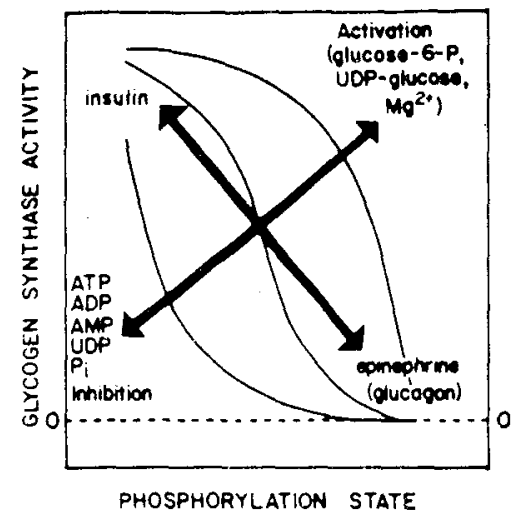

Fig. 6. Model for the interaction of hormonal and small molecule effectors in the regulation of glycogen synthase.

The diagram shows schematically a summary of the small molecule effects on glycogen synthase found here in relation to the phosphorylation state of the enzyme. Effectors are considered to alter the position of the enzyme activity-phosphorylation curves in the sense indicated. The effect of hormones, as far as this enzyme is concerned, were considered to act through phosphorylation causing progress along the activity-phosphorylation curve as indicated.

Reproduced from Ref. 9). 
the allosteric activators from the specific allosteric sites. In contrast, the active forms of the two enzymmes are able to "rise above" the cell milieu; they are not so sensitive to it and are fully active even in its presence. The covalent change in the enzyme therefore modulates the sensitivity of the enzyme to the allosteric controlling agents with a predictable pattern. The two types of control are in fact mutually interdependent as shown in Fig. 6 for the case of glycogen synthase. ${ }^{9}$ Here are plotted the interrelated effects of covalent phosphorylation control and control by allosteric activators and inhibitors. As the amount of phosphorylation increases, the enzyme activity decreases according to the 3 solid lines. Hormones such as epinephrine and glucagon, or insulin alter phosphorylation state of the enzyme. Insulin causes dephosphorylation, thus activating the enzyme while epinephrine and glucagon cause increased phosphorylation thus inactivating the enzyme. With only hormonal changes in phosphorylation state one would move up and down the central solid line. Allosteric stimulators such as glucose 6-P or others such as UDP glucose, or $\mathrm{Mg}^{2+}$ increase the activity and shift it to the upper solid curve. This is also affected by covalent phosphorylation brought about by hormones in a similar manner. Conversely alloste- ric inhibitors such as ATP, ADP, AMP, UDP, $P_{i}$ move the activity to the lower solid curve which is also influenced by covalent phosphorylation state again in a similar manner. Thus we have seen how the active forms of both enzymes are not influenced by the soluble factors in the cell and can catalyze synthesis or degradation respectively. The inactive forms on the other hand, are inactive in the cell milieu. We have also seen how the covalent and allosteric controls are mutually interacting in the case of glycogen synthase to provide a finely tuned integrated type of control.

\section{CHEMISTRY OF PHOSPHORYLATION SITES OF GLYCOGEN SYNTHASE AND PHOSPHORYLASE}

A major difference exists between glycogen synthase and phosphorylase in the detailed chemistry of the phosphorylation of the polypeptide chains which helps explain the fine tuning mechanism just discussed. Both enzymes exist normally as catalytically active dimers made up of two identical inactive monomers. The monomer of phosphorylase with a molecular weight of 94,000 daltons has a single serine phosphorylation site located near the $\mathrm{N}$ terminal end of the molecule. A single kinase, phos-

\section{MUSCLE GLYCOGEN SYNTHASE}

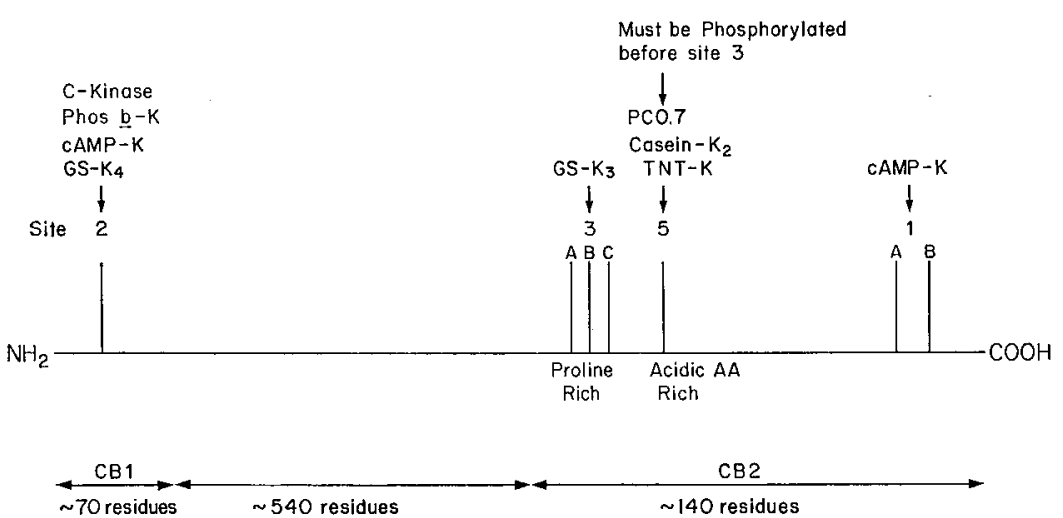

Fig. 7. Phosphorylation sites of glycogen synthase.

Sites numbered $1 \mathrm{~A}$ and $1 \mathrm{~B}$ are phosphorylated by the cAMP-dependent protein kinase; site 2 by cAMP-dependent protein kinase, phosphorylase $b$ kinase, C-kinase ( $\mathrm{Ca}^{2+}$, phospholipid, diacyl glycerol sensitive), and by glycogen synthase kinase 4 ; sites $3 \mathrm{~A}, 3 \mathrm{~B}$, and $3 \mathrm{C}$ by glycogen synthase kinase 3 ; and site 5 by troponin $\mathrm{T}$ kinase also known as casein kinase 2 or as PC 0.7 . 
phorylase $b$ kinase is responsible for catalyzing the phosphorylation at this site. A phosphoprotein phosphatase, phosphorylase phosphatase catalyzes the hydrolysis of phosphate from this site.

In contrast, the monomer of glycogen synthase with a molecular weight of 85,000 daltons has 7 serine phosphorylation sites present throughout the molecule. As shown in Fig. 7, sites $1 \mathrm{~A}$ and $1 \mathrm{~B}$ located near the $\mathrm{C}$ terminal end of the molecule are phosphorylated by the cyclic AMP-dependent protein kinase which is also able to phosphorylate site 2 at the $\mathrm{N}$ terminal end of the molecule. This same site 2 can also be phosphorylated by phosphorylase $b$ kinase, (Phos $b$-K) a $\mathrm{Ca}^{2+}$ sensitive kinase, and by the C-kinase discovered by Nishizuka and his coworkers in Japan, which is sensitive to $\mathrm{Ca}^{2+}$, phospholipids and diacyl glycerol, and by a kinase termed glycogen synthase kinase 4 (GS$\mathrm{K}_{4}$ ). In the central portion of the molecule a cluster of 3 sites termed $3 \mathrm{~A}, 3 \mathrm{~B}$ and $3 \mathrm{C}$ are phosphorylated by a kinase termed glycogen synthase kinase $3\left(\mathrm{GS}-\mathrm{K}_{3}\right)$. An additional site, 5 , is phosphorylated by a kinase termed troponin $\mathrm{T}$ kinase (TNT-K) also known as casein kinase 2, or as PC 0.7. Phosphorylation at site 5 must proceed phosphorylation at site 3. These sites are dephosphorylated by one or more phosphatases termed glycogen synthase phosphatase. Thus in contrast to phos. phorylase with one phosphorylation site and one kinase, glycogen synthase is more richly endowed with multiple phosphorylation sites and multiple kinases. These kinases are of interest in that several have known allosteric regulators which are under hormonal control. For example, cAMP activates the cAMP. dependent protein kinase and $\mathrm{Ca}^{2+}$ activates phosphorylase $b$ kinase. The C-kinase of Nishizuka, and coworkers is also of great interest since it is activated by the tumor promoter phorbol ester and when present in the cell membrane is probably identical with the phorbol ester receptor. These kinases are important links in the hormonal control which we will discuss in the next section of this paper. At present it is important to note that glycogen synthase contrasts with phosphorylase in having a multiplicity of phosphorylation sites in the molecule and a set of kinases which are themselves controlled by a number of hormonal mediators or second messengers. Glycogen synthase itself, like UDPG is therefore a marvellous development of nature compared to phosphorylase, and is programmed like a computer for finely tuned control. Thus, glycogen synthase is more highly developed both as regard to its function and for its control than is phosphorylase. Glycogen synthase can be thought of in terms of a rheostat control compared to phosphorylase which can be thought of in terms of an on-off switch.

\section{HORMONAL CONTROL}

As already mentioned, hormonal control of glycogen synthase and phosphorylase is a very important mechanism. In general the major hormonal controls are exerted via epinephrine and glucagon which increase phosphorylation state and via insulin which decreases phosphorylation state. How are these changes brought about intracellularly by these hormones which act by binding to receptors in the cell membrane? The answer lies in the use of second messengers or mediators. These molecules relay the hormonal message from the outside to the inside of the cell.

The first discovered (Rall and Sutherland) and best understood second messenger is cyclic AMP. It is the second messenger for the hormone glucagon and also for the hormone epinephrine when it acts on a specific type of adrenergic receptor termed a $\beta$ receptor. In either case, the membrane bound enzyme adenylate cyclase is activated and proceeds to produce increased cyclic AMP formed from ATP, with the other product being pyrophosphate. This is shown diagramatically in Fig. 8. The interaction of the hormone with its receptor (either, glucagon or epinephrine) leads to increased adenylate cyclase activity. The cyclase catalytic subunit is activated by an intermediately coupling subunit complex called $\mathrm{N}$ and made up of $\alpha, \beta$ and $\gamma$ subunits one of which the $\alpha$ subunit binds and hydrolyzes GTP.

A second mediator is $\mathrm{Ca}^{2+}$. When epinephrine interacts with a different receptor termed an $\alpha$ adrenergic receptor, $\mathrm{Ca}^{2+}$ enters the cell, 


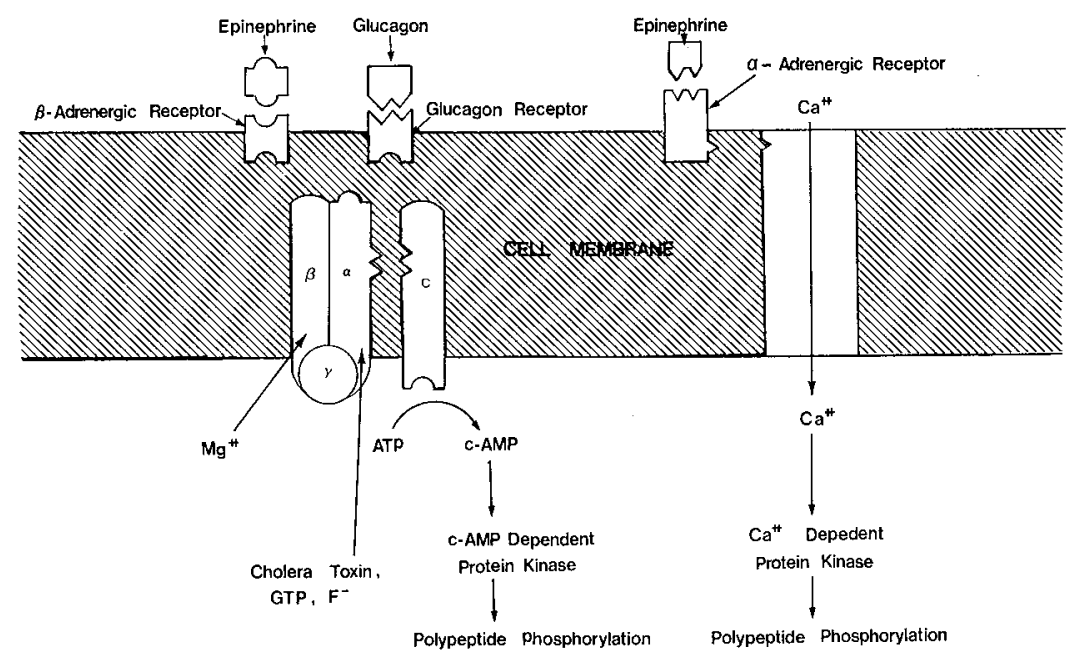

Fig. 8. Model of epinephrine and glucagon hormone action.

The cell membrane containing $\alpha$ and $\beta$ adrenergic and glucagon receptors is shown. The adrenergic and glucagon receptors are coupled to adenylate cyclase, the adrenergic receptor is coupled to a $\mathrm{Ca}^{2+}$ channel. Activation of adenylate cyclase catalytic subunit occurs via a receptor ligand activation of the intermediate coupling subunit leading to a dissociation of $\alpha$ and its activation via GTP binding and hydrolysis. $\alpha$ can also be activated by $\mathrm{F}^{-}$and by cholera toxin which acts to ADP ribosylate. Activated catalytic subunit $C$, then catalyzes the formation of cAMP. Both second messengers cAMP, and $\mathrm{Ca}^{2+}$ stimulate polypeptide phosphorylation of phosphorylase and glycogen synthase.

increases in the cytoplasm and becomes a second messenger.

How do cAMP and $\mathrm{Ca}^{2+}$ act as second messengers to increase the phosphorylation state of the enzymes phosphorylase and glycogen synthase? Both second messengers activate specific kinases or phosphotransferases which then phosphorylate phosphorylase and glycogen synthase. Thus, cAMP-dependent protein kinase is activated by cyclic AMP and phosphorylase $b$ kinase is activated by $\mathrm{Ca}^{2+}$. There are specific binding subunits in these enzymes which bind cyclic AMP and $\mathrm{Ca}^{2+}$ respectively and thus recognize these second messengers. Now, we can understand how, via these 2 second messengers the increased phosphorylation states of the 2 enzymes phosphorylase and glycogen synthase are obtained. This result profoundly alters their activity in the cell as described in the previous section.

With insulin, which causes dephosphorylation, a similar mechanism exists. But a different second messenger or set of messengers are used.
We have been studying this problem by isolating the insulin second messengers for the past 5 years and now have evidence that they are oligopeptides or oligoglycopeptides. We think there may be several formed and not just one.

To control dephosphorylation of glycogen synthase, we have obtained evidence that there are two messengers. One inhibits the cyclic AMP-dependent protein kinase. ${ }^{10)}$ The action of this mediator is shown in Fig. 9. It inhibits the enzyme in the presence of low concentrations of cyclic AMP. As the concentration of cyclic AMP is increased, the inhibition is partially but not completely overcome. A second mediator peptide is also formed by insulin action which stimulates the phosphoprotein phosphatase which dephosphorylates glycogen synthase. Its action is shown in Fig. 10. Thus insulin acts to decrease phosphorylation of glycogen synthase by inhibiting the kinase and by stimulating the phosphatase. We think insulin acts by a novel mechanism to form several mediator 
peptides by stimulating their formation proteolytically from a precursor glycoprotein present in the cell membrane.

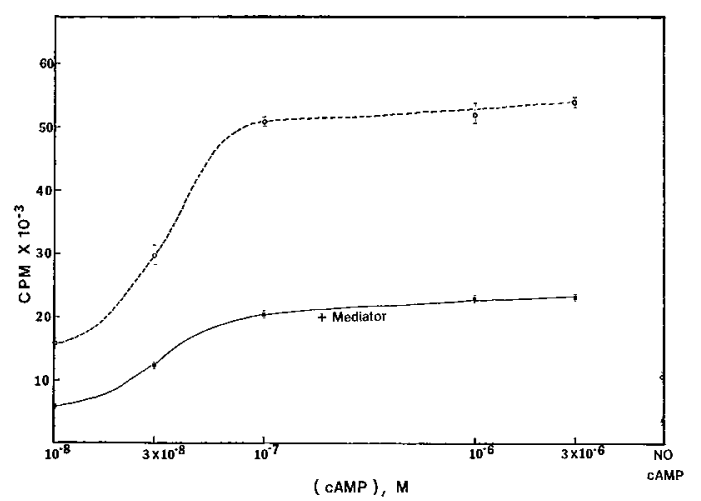

Fig. 9. Effect of insulin mediator on activation of cAMP-dependent protein kinase by cAMP.

$0.5 \mathrm{mg} / \mathrm{ml}$ histone II A, $10 \mathrm{mM} \mathrm{MgCl}_{2}, 50 \mathrm{mM}$ MES pH 6.5, $60 \mu \mathrm{M}{ }^{32} \mathrm{P}-\mathrm{ATP}$ and the various concentrations of $\mathrm{cAMP}$ as indicated were incubated with enzyme at $30^{\circ} \mathrm{C}$ for $10 \mathrm{~min}$ in a total volume of $100 \mu 1$. At the end of the incubation, $75 \mu 1$ aliquots were spotted on Whatman 3 paper squares $(2 \times 2 \mathrm{~cm})$ and immersed in 10\% TCA $-50 \mathrm{mM} \mathrm{H} \mathrm{H}_{3} \mathrm{PO}_{4}$. Papers were washed and radioactivity counted. Each point represents the average of duplicate determinations. Mediator concentration was $6 \times 10^{-7} \mathrm{M}$.

Reproduced from C. VILlaR-PALASI, L. C. HUANG and J. LARNER (unpublished).

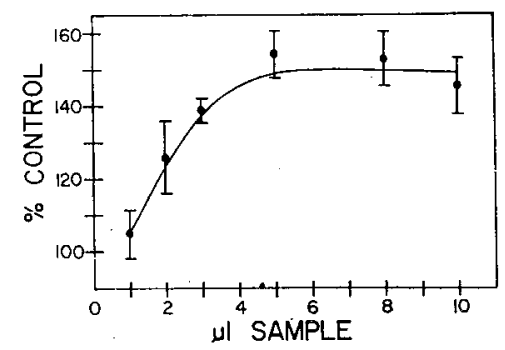

Fig. 10. Dose-response curve of insulin mediator on type 1 protein phosphatase.

Increasing volumes of pooled, lyophilized saline, and insulin-treated Sephadex G-25 chromatographed extracts were assayed with type 1 protein phosphatase. Each point is the mean of five determinations with the indicated standard deviations.

Reproduced from Ref. 10).

\section{SUMMARY}

I have now taken you over some 35 years of research to the forefront of our research in this area at the present time. I hope I have given you an overall picture of this fascinating area of metabolism which is under such very elegant control by interrelated covalent and non-covalent mechanisms.

Our own work has led to the discovery of the action of the debranching enzyme amylo1,6-glucosidase, the mechanism of action of the branching enzyme amylo- $1,4 \rightarrow 1,6$-transglucosidase, the discovery of the covalent control of glycogen synthase by the multiple phosphorylation of glycogen synthase on a single polypeptide chain, the discovery of the mechanism of action of insulin to bring about the activation of glycogen synthase by dephosphorylation, the discovery of the inactivation of the cAMPdependent protein kinase by insulin and most recently the discovery of the novel insulin second messenger peptides. I wish to acknowledge the work of many coworkers in my own laboratory and the scientists in many other laboratories throughout the world who have also contributed to our understanding of this field of research.

At present it is clear that covalent control by phosphorylation of key catalysts within the cell is a dominant theme for research in the mechanism of hormonal control. Perhaps similar mechanisms of action may apply in the case of plant cells as well. The late Dr. Earl Sutherland, one of my teachers, told me in his last conversation with me just before he died, that he was going to begin research in the mechanism of action of plant hormones.

\section{REFERENCES}

1) G. T. Cori and J. LaRner: J. Biol. Chem., 188, 17 (1951).

2) J. LARner, B. Illingworth, G. T. CoRi and C. F. CORI: J. Biol. Chem., 199, 641 (1952).

3) J. LARNER, Y. TAKEDA and S. HizUKURI: Mol. Cell. Biochem., 12, 131 (1976).

4) S. HizuKuri, T. KANEKo and Y. TAKedA: Biochim. Biophys. Acta, 760, 188 (1983).

5) C. Villar-Palasi and J. LARner: Arch. Biochem. Biophys., 86, 61 (1960). 
6) L. F. Leloir, J. M. Olavarria, S. H. GOLdenBERG and H. CARMINATTI: Arch. Biochim. Biophys., 81, 508 (1959).

7) J. LARNER: J. Biol. Chem., 202, 491 (1953).

8) J. LARNER: Circ. Res., 38, I-2 (1976).

9) P. J. RoACH and J. LARNer: J. Biol.Chem., 251,
1920 (1976).

10) K. Cheng, M. Thompson, C. Schwartz, C. MALCHOFF, S. TAMURA, J. CRAIG, E. LOCKER and J. LARNER: in Molecular Basis of Insulin Action, M. P. CzECH, ed., Plenum Press., New York, p. 171(1985). 